\title{
A review on massive e-learning (MOOC) design, delivery and assessment
}

\author{
Thanasis Daradoumis ${ }^{1,2}$ \\ Roxana Bassi \\ Fatos Xhafa ${ }^{2}$ \\ adaradoumis@uoc.edu \\ rox@roxanabassi.com.ar \\ fxhafa@uoc.edu \\ Santi Caballé ${ }^{2}$ \\ scaballe@uoc.edu
${ }^{1}$ Department of Cultural Technology and Communication (D.C.T.C), School of Social Sciences, University of Aegean, Mytilini, Greece
${ }^{2}$ Department of Computer Science, Multimedia and Telecommunications, Open University of Catalonia, Barcelona, Spain

\begin{abstract}
MOOCs or Massive Online Open Courses based on Open Educational Resources (OER) might be one of the most versatile ways to offer access to quality education, especially for those residing in far or disadvantaged areas. This article analyzes the state of the art on MOOCs, exploring open research questions and setting interesting topics and goals for further research. Finally, it proposes a framework that includes the use of software agents with the aim to improve and personalize management, delivery, efficiency and evaluation of massive online courses on an individual level basis.
\end{abstract}

Keywords: $M O O C$, OER, agents, e-learning, virtual learning environment (VLE)

\section{INTRODUCTION}

UNESCO considers that universal access to high quality education is the key to the building of peace, sustainable social and economic development, and intercultural dialogue. Open Educational Resources (OER) provide a strategic opportunity to improve the quality of education as well as facilitate policy dialogue, knowledge sharing and capacity building. [1]. A recent popular trend in e-learning are MOOCs or Massive Online Open Courses. According to Siemens [2] MOOCs are a continuation of the trend in innovation, experimentation and the use of technology initiated by distance and online learning, to provide learning opportunities for large numbers of learners. The Commonwealth of Learning [3] indicates that MOOCs are a means of facilitating the efficient creation, distribution and use of knowledge and information for learning by taking advantage of freely available online resources such as OER, and that they can be used to support social networking and other forms of "connectivity" among the participants. Therefore the power of the MOOC is based on the active engagement of large numbers of self-organizing learners and the connections they build amongst them using the course platform and other available social interaction tools.

In the first part of this paper (Section II) we develop an analysis of the state of the art on MOOCs in the areas of design, delivery and assessment. In Section III, we set up the context of our research by identifying issues related to improved and personalized management, delivery and assessment of massive online courses. In particular, we define our research problem, which we break down into specific research topics. We then analyze the topics into open research questions and we establish our research goals. In Section IV, we contribute our own proposal for improving
MOOC design, delivery and assessment using software agents.

\section{STATE OF THE ART ON MOOCS}

The first open e-learning course for a large number of participants was launched about five years ago [2], but the concept became widespread in 2012 when several large Universities started their own MOOCs. According to Siemens [2] open online courses, sometimes called "massive" (MOOCs) due to their high enrolment numbers, offer a middle ground for teaching and learning between the highly organized and structured classroom environment and the chaotic open web of fragmented information. Therefore MOOCs have characteristic - and challenges - of its own, due mostly to the large number of simultaneous participants, with some courses offered by Coursera and Udacity exceeding 100,000 registrants [2].

Based on research work conducted so far, in this section we present some of the distinctive features of the design, delivery and assessment of MOOCs, and discuss the main challenges in each of these areas.

\section{A. Design}

MOOC models are evolving quickly, according to Siemens [2] they can be currently classified as xMOOCs, cMOOCs and quasi-MOOCs. xMOOCs replicate online the traditional model of an expert tutor and learners as knowledge consumers, with saved video tutorials and graded assignments. cMOOCs are based on a connectivist pedagogical model that views knowledge as a networked state and learning as the process of generating those networks, in this case using online and social tools. Lastly, the category of quasi-MOOCs encompasses a myriad of web-based tutorials as OER that are technically not courses but are intended to support learning-specific tasks and consist of asynchronous learning resources that do not offer the social interaction of cMOOCs or the automated grading and tutorial-driven format of xMOOCs.

Although many MOOC formats exist, according to Glance et al. [4] who made a sample of them in 2012, most courses exhibit common defining characteristics that include massive participation, online and open access, lectures formatted as short videos combined with formative quizzes, automated assessment and/or peer and self-assessment, and online forums and applications for peer support and discussion. MOOC design should favor a Learner-Centered 
Approach, providing strategies that change the perception of learners as active participants in the establishment of individual goals and a personal trajectory [5]. Therefore it would be desirable that MOOCs allow some degree of content customization based on user profiling that optimizes the learning process.

\section{B. Delivery}

MOOCs typically involve a very large number of participants, making the management of courses quite a complex issue. Massive also raises concerns about isolation and overwhelming student-instructor ratios [2]. Because of sheer numbers, tutor involvement during delivery stages has to be limited to the most critical tasks. Tutoring is usually and consequently poor, since minimal feedback is received by the participants and peer-based evaluation is valuable but often unprofessional and lacking the necessary expertise, both didactical and on the specific subject.

MOOCs are intended for thousands of simultaneous participants from different origins and with different backgrounds, education levels and language proficiencies. Data stored at the LMS (Learning Management System) during course access related to individuals' online behavior can provide insight into student learning processes and the relationship between online trace data and academic performance alerting educators to when intervention may be required or if their learning activities may need to be adjusted. [6].

Managing course delivery for thousands of participants at the same time requires completely adapting the traditional tutor-assisted e-learning model, and calls for the use of automated tools that can complement the lack of human attention while maintaining acceptable quality standards.

\section{Assessment}

A prominent feature of running a course with large numbers of learners is the impossibility of providing marks and feedback that is not either automated or peer assessed [4]. In MOOCs it is not possible for human tutors to follow up with every student and review and grade assignments individually, whereas the design must facilitate large-scale feedback and interaction. In order to meet the challenges of large numbers of learners, assignments are computer-graded in XMOOCs [2]. But computer-based grading is many times limited, disappointing and insufficient, with no partial marks and detailed explanations of answers.

In e-learning environments learners would also be interested in knowing whether they are more likely to succeed or fail towards reaching their educational goals (self-regulated learning), and would like to receive more feedback elements besides formal assessments. It also happens that the resources of the participatory method that are a hallmark of MOOCS have to be limited while testing, and test content must be changed with every cohort in order to reduce cheating, imposing additional workload on course designers and tutors. At the same time, the lack of user authentication cannot ensure the real identity of the MOOC participant, which should be verified in order to satisfy accreditation.

\section{Summary of challenges}

Next, we present a summary of key challenges that MOOCs designers and providers face and which need further research.

Summary of key challenges for MOOCs providers:

- Among the key design characteristics of MOOCs, massiveness and low teaching involvement during delivery stages have been identified as the biggest challenges for MOOC design [5].

- Since it is recognized that the heterogeneity of MOOC learners is high, and their level of maturity and experience is also varied, courses have to be conceptualized taking into account a large number of simultaneous participants with different educational and cultural backgrounds. Currently content is delivered to learners regardless of their differences in performance and behavior in the environment. But unless content could be customized and personalized according to profile characteristics, it could result in a frustrating learning environment for participants because it lacks adaptability to their specific needs and learning styles.

- One key concern of MOOCs is a high learners' dropout rate, with several sources indicating that about $5 \%$ to $15 \%$ of participants finish the courses on average. However, some authors suggest that dropout statistics might not be representing the only reality of MOOC learners. This is because different patterns of student behavior exist, and analyzing further each participant's objectives can provide additional insight into the different personal goals when attending a course besides finalizing it. Therefore different measures should be available in order to evaluate whether MOOCs are effective for individual participants [7][8].

- MOOCs offer particular challenges for what is becoming accepted as mainstream practice in learning analytics. Because of the relative newness of MOOCs as a widespread phenomenon, there is not yet a substantial body of literature on the learning analytics of MOOCs [9]. Data analysis is currently very limited, as existing MOOC platforms offer limited data storage and analysis, whereas usability to analyze this data is low.

- Testing options are limited because assessments have to be conducted using automated tools and allowing limited peer interaction. Tests have to be changed for every cohort to avoid cheating.

\section{SETTING UP THE CONTEXT OF OUR RESEARCH}

MOOCs offer opportunities and pose challenges never faced before, simply because until not long ago it was unthinkable to have several thousand individuals participate 
simultaneously in an online course. But as UNESCO states, the chance of offering access to quality education to every individual that can be connected is an opportunity that cannot be wasted. We believe that taking into account the design, delivery and assessment characteristics and constraints of MOOCs, more research is needed on the potential use of automated tools like agents to overcome some of the challenges that have been identified, as well as optimize efficiency, reduce costs and improve the learning process. According to MIT software agents group, agents are "computer systems to which one can delegate tasks. Software agents differ from conventional software in that they are long-lived, semi-autonomous, proactive, and adaptive". Software agents can use artificial intelligence methods in order to analyze information and react to it. But there are also simpler agents that are not necessarily intelligent, and the ability to incorporate a non-intelligent agent into a multimedia learning environment with relative ease may increase the effectiveness of the environment for minimal cost [10].

\section{A. Use of agents in educational environments}

The opportunities for using agents in e-learning courses are enormous. Agent characteristics like autonomy, abilities to perceive, reason and act in specialized domains, as well as their capability to cooperate with other agents makes them ideal for e-learning applications [11]. No research has been reported so far for the use of agents in the management or delivery of MOOCs. However, agents have been widely used in educational environments, first in Intelligent Tutoring Systems (ITS) and lately in Virtual Learning Environments (VLE). Agent use in e-learning environments has been researched many years before the advent of MOOCs, defining some potential roles for them like for example Pedagogical Agents (tutor, mentor, assistant), Web Agents (working with Internet applications and social networking tools), Learner's agents and mixed agents which could teach and learn [12].

Intelligent tutoring systems use agents designed to transmit specific knowledge to students by an interactive individualized process that emulates a human tutor guiding a student in his or her learning process, and could adapt the content to the participant's needs, profile and learning style [13].

Pedagogical Agents have been used as learning aids, many times taking the form of animated figures, so that learners might perceive the agent interaction as a social exchange [14]. Recent implementations suggest that while it previously may have taken a considerable amount of time and resources to design and implement a pedagogical agent within a learning environment, advances in technology have made pedagogical agent-based systems more accessible and affordable to educators [10].

Recommender agents are able to adapt course content to the learners profile using web mining techniques [15]. Recent research indicates that this customization should consider the learner's profile, the learner's knowledge and learner's educational preferences. All these components should be detected automatically within e-learning systems [16].

We conclude by stating that in MOOC environments, agents could be used to analyze data produced by the MOOC platform, systems and participants, and use it intelligently or mechanically to improve design, delivery and assessment.

\section{B. Research problem and topics}

The main research question we ask is the following: How could software agents be used to optimize and personalize delivery, management and assessment of massive online courses (MOOC)?

This research problem is further broken down into the following research topics and research questions concerning the areas of MOOC course design, delivery and assessment.

C. Design

- How could data collected by agents provide feedback on course design? How could collected information help us understand better how learning styles might affect the successful progression of MOOCs' learning experience?

- How could we better identify technical challenges that frustrate or demotivate participants? (i.e. technologies, clients, data formats)

D. Delivery

- How could agents offer support to adapt course content in order to offer a personalized learning experience for different participant profiles?

- How could agents be used to detect usage patterns that can, for example, be used for early detection of potential drop outs? How could agents assist tutors in their daily tasks? How could agents automate routine tasks through alert systems?

- How could we use agents in order to optimize the learning analytics of MOOCs? Which indicators should we measure in MOOCs according to different stakeholders? Which are the best analysis methods and ways to represent and show the results? How could the collected data be used to improve course content?

\section{E. Assessment}

- How could agents assist in building a robust automated assessment methodology by integrating assessment techniques such as student e-portfolio, learning analytics and enriched rubrics (including self assessment rubrics and peer assessment rubrics)?

- How could user profile and personalization improve the evaluation methods, adjusting them according to each participant's individual educational objectives 
and learning style? How could agents use the data produced from learning analytics of MOOCs in order to assess and monitor learners' learning process and performance in a more efficient manner?

- How could agents be used during assessment in order to verify the student's identity and minimize cheating and fraud?

In the following section we present a framework on how software agents could be applied to answering these questions.

\section{PROPOSED FRAMEWORK: AGENTS IN MOOCS}

In this section we will describe an agent-based framework that could provide improved and personalized management, delivery and assessment of massive online courses.

Acting as intermediaries between the MOOC learning environment and different stakeholders, agents can serve the different informational needs and use their intelligence to detect situations that need intervention or alerts. Agents can perform a data mining analysis on the data the MOOC system (Learning Management System or LMS) and other related applications like external databases, social network tools, etc. store. Collected data can then be analyzed from several perspectives (educational, pedagogical, content quality, student performance, usage parameters, user profiling, time management etc.) in order to provide different stakeholders with information for improving the efficiency and effectiveness of the e-learning activities. Agents could also execute automated tasks, like send alerts to tutors and reminders to learners, or serve customized content pages, according to pre-programmed behavioral scenarios.

Figure 1 presents an agent-based framework that provides an infrastructure to effectively support MOOCs management at every stage (design, delivery and assessment).

Next, we provide some examples of how agents could be used to support course design, delivery, assessment, and enhanced learning analytics.

\section{A. Agents in MOOC Design}

Using their abilities agents could perform data interpretation that is not available on standard MOOC applications. By analyzing the MOOC environment and other related system's data, agents could provide information on course usage like for example usage patterns, navigation, content sequencing, problematic content areas or data formats, tool usage and student's profiling. This information could in turn provide feedback to course designers in order to improve MOOC usage for future cohorts.

Agents could also allow for a more learner-centered approach by providing customized content according to the learners profile and needs. This is explained in more detail below.

\section{B. Agents in MOOC delivery}

\section{1) Content customization}

Modern learners are interested in optimizing the time spent in learning activities and their effectiveness with respect to their individual capabilities, expertise, preferences and learning objectives. In this regard they would like the MOOC environment to understand their learning style and adapt the learning situation in terms of specific content,

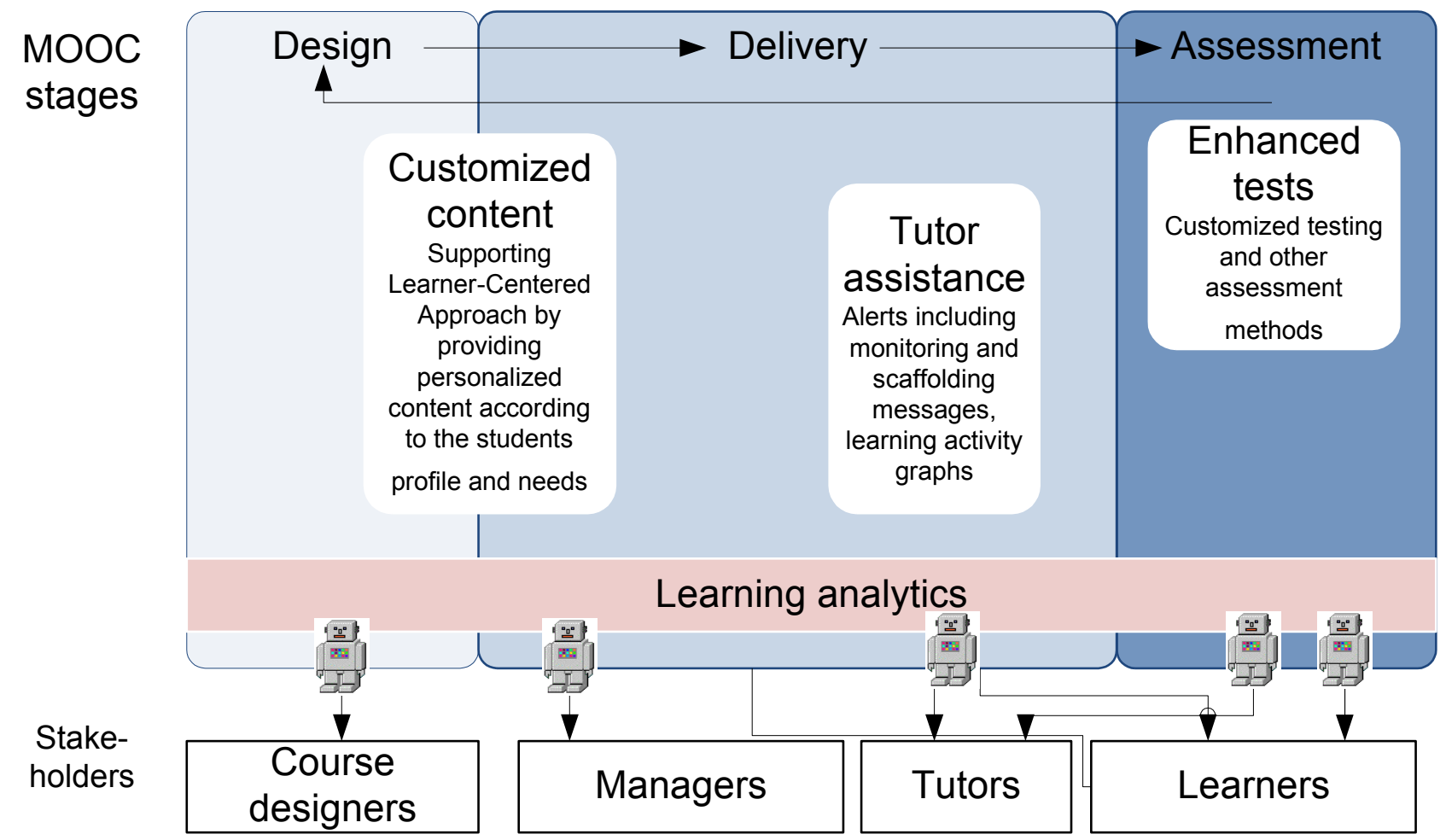

Figure 1 - An agent-based framework that provides an infrastructure for supporting MOOCs stages effectively. 
didactic approaches, the type of media to be used, the way concepts to learn are sequenced and so forth, while getting adaptive/personalized feedback for improving their performance and their motivation as well.

A recommender system in an e-learning context is a software agent that tries to "intelligently" recommend actions to a learner based on the actions of previous learners [16]. Agents using learning/prediction algorithms could be used to support scenario based learning by dynamically adjusting course content to suit each participant's profile and needs, allowing for a personalized delivery of course content even for thousands of simultaneous users. This way a MOOC course could become a personal learning experience, with specific content delivered individually based on the learners learning outcomes, profile, location, behavior, interests, preferences, time limitations, etc. With the support of agents even a very large number of participants can enjoy a unique online experience that supports a quality learning process without consuming tutoring time.

For example, agents analyzing the learner's profile could customize a course as follows:

- Adjusting course content according to the participants pre-requisites or educational background

- Changing course content parameters according to the participant's location or country of origin, for example language, units of measure, currency symbol, seasons, etc.

- Showing relevant case studies or further readings according to the country or region of origin/interest

The use of automated customization could help reduce average cost per participant for large scale training, without lowering the quality

\section{2) Tutor assistance and alerting systems}

Standard e-learning courses store a large quantity of valuable data on course usage and access, though it might not always be easy for tutors to interpret it or access it when it is needed. Data analysis offered by agents could provide tutors with course real time statistical information in order to detect trends and deviations and act accordingly, focusing the use of their time. Because of the large volume of participant's collected data, MOOCs present a critical improvement from the small populations of most online courses: large populations can be used statistically to detect trends, make inferences more accurately and identify anomalies with more certainty. Human tutors might not have the time to follow up with individual participants and their learning curves, but because MOOCs have a large population of learners -statistically speaking - agents could alert tutors about anomalies, for example the extremes of the Gaussian curve of the population for any given parameter, people doing extremely below or above average, and detect potential drop-outs, problems, cheating or outstanding learners.
The collected data can be extremely valuable in order to provide useful information to improve the design and delivery of online courses, customize the learning experience, adjust learning times according to profiling, make a better use of limited human resources and detect loss of interest and potential drop-outs before they occur.

\section{Agents in MOOC assessment}

Agents could be used to improve current automated test by adjusting assignment questions according to the participant's educational level, or changing the sequencing of the evaluation questions if participants fail or pass a certain question before that one. Moreover, agents could assist in building a robust automated assessment methodology by integrating assessment techniques such as student e-portfolio, learning analytics and enriched rubrics (including self assessment rubrics and peer assessment rubrics).

Finally, agents could use the data produced from learning analytics of MOOCs in order to assess and monitor learners' learning process and performance in a more efficient manner. It is hypothesized that if the learner perceives the computer interaction as social communication, it may cause increased performance on transfer tests due to the student engaging in the "sense-making process" [14].

Agent intelligence could also be used to further enhance user identity verification and reduce fraud and cheating during tests.

\section{Learning Analytics}

Tutors and course designers typically keep on wanting to know whether their didactic approaches are yielding positive results and would like to adjust them accordingly. At the same time, no matter how large their student base is, they would appreciate knowing who their learners are in terms of identity, profile, preferences, and learning style, as well as to continuously and effectively monitor how they are faring over time throughout their learning progression.

Course designers, managers, tutors, participants and policy-makers of educational institutions might benefit from harnessing all the data MOOCs collect, and use them for improving educational activities, courses delivered, the learning experience as a whole and the investments of entire educational offers.

Software agents could be designed to collect data automatically from the e-learning environment according to pre-defined indicators contained in a framework using advanced Educational Data Mining and Learning Analytics techniques and tools. From this data, for example, course administrators and managers could learn of usage profile and access times, study patterns, problems accessing a resource, detect learning content that is never accessed or too much accessed. Tutors could detect trends and be alerted when their involvement is required. Learners could be 
automatically alerted about missing task completion, deadlines, deviations from the norm, etc. Decision makers could also receive information that allows them to analyze the cost/effectiveness ratio of the courses, measure the quality of the learning offering provided, predict success/failure and drop-out rate of their learners and adjust their learning offer accordingly.

In sum, considering there is not yet a substantial body of literature on the learning analytics of MOOCs [9], an effective collection and analysis of the large volume of data coming from MOOCs and related systems, as well as the use of the outcome of appropriate modeling and reasoning activities upon such data becomes an imperative need. This can not only improve the use of existing systems, but also pave the way for improving future versions of MOOCs, both from the tutor's and learners' point of view, as well as providing decision makers with innovative means for structuring and updating the educational offer they are responsible of within their institutions.

\section{CONCLUSIONS}

The four agent-provided services presented are just an example of services agents and agent swarms could provide in order to improve efficiency in MOOCs. We believe MOOCs offer a remarkable opportunity for the training of thousands of participants distributed all over the world, allowing online access to quality education through a new way of learning. MOOCs are a recent trend in e-learning; their structure and format is being constantly adapted as more experience is gained with their delivery and so it is important to understand their benefits and shortfalls in a systematic manner [4].

Data collected from MOOC environments with the assistance of agents could be used to improve design, delivery and assessment. Learning analytics can also provide invaluable data on learning profiles of diverse participants. So far, existing MOOC environments have shared little information on their usage patterns. We hope that with the inclusion of software agents MOOC can experience noticeable improvements in content quality, course delivery, less drop-out rates, better participant support and enhanced evaluation methods.

It would also be interesting to determine how agent swarms, using adequate knowledge representation, could share collected information about the MOOC environment and react automatically to stimulus.

We believe that given the large number of students and student generated data, as well as the limited human resources available, agents are not a desirable characteristic but a necessity for future MOOC applications to be successful.

\section{REFERENCES}

[1] United Nations UNESCO, "Paris OER Declaration", June 2012, Retrieved from: http://www.unesco.org/new/fileadmin/MULTIMEDIA/HQ/CI/WPFD 2009/English_Declaration.html on 15/3/2013.
[2] G. Siemens "Massive Open Online Courses: Innovation in Education?", eds. Commonwealth of learning, Perspectives on Open and Distance Learning: Open Educational Resources: Innovation, Research and Practice, p. 5. 2013, retrieved from http://www.col.org/resources/publications/Pages/detail.aspx?PID=44 6 on $21 / 5 / 13$

[3] Commonwealth of Learning (COL), "Perspectives on Open and Distance Learning: Open Educational Resources: Innovation, Research and Practice", May 2013, retrieved from http://www.col.org/resources/publications/Pages/detail.aspx?PID=44 6 on $21 / 5 / 13$

[4] D. Glance, M. Forsey, M. Riley M., "The pedagogical foundation of massive online courses", 2012, First Monday Journal, retrieved from http://firstmonday.org/ojs/index.php/fm/article/view/4350/3673 on 12/6/13, DOI: http://dx.doi.org/10.5210\%2Ffm.v18i5.4350

[5] L. Guàrdia, M. Maina, A. Sangrá A, "MOOC Design Principles. A Pedagogical Approach from the Learner's Perspective", May 2013, retrieved from http://elearningeuropa.info/sites/default/files/asset/Indepth_33_4_0.pdf on 21/6/13

[6] N. Mirriahi, S. Dawson S., "The Pairing of Lecture Recording Data with Assessment Scores: A Method of Discovering Pedagogical Impact", 2013, doi: 10.1145/2460296.2460331

[7] P. Hill, "Emerging Student Patterns in MOOCs: A (Revised) Graphical View", 2013, online blog post retrieved from http://mfeldstein.com/emerging-student-patterns-in-moocs-a-revisedgraphical-view/ on 12/6/13

[8] R. Kizilcec, C. Piech. E.Schneider, "Deconstructing Disengagement: Analyzing Learner Subpopulations in Massive Open Online Courses", 2013, retrieved from http://www.stanford.edu/ cpiech/bio/papers/deconstructingDisengage ment.pdf on 26/6/2013, doi:10.1145/2460296.2460330

[9] D. Clow, "MOOCs and the funnel of participation", Third Conference on Learning Analytics and Knowledge, 2013, retrieved from http://oro.open.ac.uk/36657/1/DougClow-LAK13-revisedsubmitted.pdf on 21/6/2013, doi:10.1145/2460296.2460332

[10] N. Schroeder, O. Adesope, "A Case for the use of Pedagogical Agents in Online Learning Environments", 2012, retrieved from http://jotlt.indiana.edu/article/view/2126/3045 on 2/6/2013

[11] M.P. Papazoglou, "Agent-oriented technology in support of ebusiness", 2001, Communications of the ACM, Vol. 44 No. 4, pp. 717.

[12] L.M. Martins Giraffa and R.M. Vicari Rosa "The Use of Agents Techniques on Intelligent Tutoring Systems" 1998, retrieved from http://lsm.dei.uc.pt/ribie/docfiles/txt200342413856156.PDF on $3 / 5 / 2013$

[13] J. Gascueña, A. Fernández-Caballero, “An Agent-Based Intelligent Tutoring System for Enhancing E-Learning / E-Teaching”, 2005, retrieved

http://www.itdl.org/journal/Nov_05/Nov_05.pdf\#page=15 on $13 / 3 / 2013$

[14] R.E. Mayer, S.C. DaPra, "An embodiment effect in computer-based learning with animated pedagogical agents", 2003, Journal of Experimental Psychology: Applied, 3, 239-252.

[15] O. Za1ane "Building a Recommender Agent for e-Learning Systems", 2002 , retrieved

from http://scholar.google.com.ar/scholar_url?hl=es\&q=http://citeseerx.ist. psu.edu/viewdoc/download\%3Fdoi\%3D10.1.1.123.1521\%26rep\%3D rep 1\%26type\%3Dpdf\&sa=X\&scisig=AAGBfm2Rgu4GpP9aXE6zJD 3UKXCupVJmjA\&oi=scholarr\&ei=ke7jUb6SDcHoswbJ8IDwAg\&v ed $=0$ CDwQgAMoADAA on 3/6/2013

[16] M.K. Khribi, M. Jemni, O. Nasraoui, "Automatic Recommendations for E-Learning Personalization Based on Web", 2009, Usage Mining Techniques and Information Retrieval. Educational Technology \& Society, $12 \quad$ (4), 30-42. retrieved from http://www.ifets.info/journals/12_4/4.pdf on 3/6/2013 